\title{
Editorial
}

\section{Long life to “The European Physical Journal - APPLIED PHYSICS"}

We are happy to introduce you the first issue of the new "The European Physical Journal - Applied Physics". The aim of this initiative, which concretises the merging of two well-known French journals, the Journal de Physique III and Microscopy, Microanalysis, Microstructures, is to initiate within a European perspective the basis of an international journal of Applied Physics. This creation accompanies the broader movement opened with the merging of the more than centennial publications namely, Journal de Physique and Zeitschrift für Physik, in order to give birth to an attractive forum for all those involved in this field of activity, in the European physics community evidently but also all around the world. This journal covers a wide range of domains in materials science, optics, electronics and instrumentation in general: a more comprehensive list of topics is given on page A6.

The journal is represented in a large number of countries thanks to our team of Associate Editors working in close collaboration with the central editorial offices: the one in Paris at the headquarters of the French Society of Microscopies is more specific for the authors submitting manuscripts involving all types of microscopies and analysis techniques together with their use for materials characterization, while the Orsay office is intended to process all the other types of papers. However you can also submit your manuscripts directly to any of the Associated Editors listed on the title page of the journal. With the support of the staff of EDP Sciences we will all devote our efforts to insure fast and high quality production.

EPJ Applied Physics will appear monthly. Submission is welcome electronically as well as in paper form; see the conditions reported on the previous pages.

C. Colliex and M. Sauzade 